\title{
Caracterização do Regime de Vento no Município de Seropédica, Rio de Janeiro (2001-2010)
}

\author{
José Francisco Oliveira Júnior ${ }^{1}$, Jessica Cristina Santos Souza², \\ Flavia Oliveira Dias ${ }^{2}$, Givanildo Gois ${ }^{3}$, Itamar Frederico Souza Gonçalves ${ }^{3}$, \\ Mauricio Soares da Silva ${ }^{4}$
}

\begin{abstract}
${ }^{1}$ Departamento de Ciências Ambientais - DCA, Universidade Federal Rural do Rio de Janeiro - UFRRJ, Seropédica/RJ, Brasil ${ }^{2}$ Colégio Técnico - CTUR, Universidade Federal Rural do Rio de Janeiro - UFRRJ, Seropédica/RJ, Brasil

${ }^{3}$ Programa de Pós-graduação em Ciências Ambientais e Florestais - PPGCAF Departamento de Ciências Ambientais - DCA, Universidade Federal Rural do Rio de Janeiro - UFRRJ, Seropédica/ RJ, Brasil

${ }^{4}$ Programa de Engenharia Mecânica - PEM, Instituto Alberto Luiz Coimbra de Pós-graduação e Pesquisa de Engenharia - COPPE, Universidade Federal do Rio de Janeiro - UFRJ, Rio de Janeiro/RJ, Brasil
\end{abstract}

\begin{abstract}
RESUMO
Baseado em dez anos de dados de direção e velocidade de vento da Estação Meteorológica Automática de Superfície (EMAS), foi caracterizado o regime de vento em superfície em Seropédica, Estado do Rio de Janeiro (RJ). Foram elaborados histogramas e rosas do vento decenal, sazonal e por períodos (madrugada, manhã, tarde e noite). As direções predominantes decenais foram $\mathrm{N}$ e SW, sendo associadas ao regime de brisa de vale/montanha e brisa marítima, juntamente com a passagem de Sistemas Frontais. As classes de vento decenal foram calmaria (abaixo de $\left.1 \mathrm{~m} . \mathrm{s}^{-1}\right)(40,2 \%)$ e ventos fracos $\left(1 \mathrm{a} 3 \mathrm{~m} \cdot \mathrm{s}^{-1}\right)(39,8 \%)$. Sazonalmente, as direções predominantes foram N, NW, NNE, SSW e SW (Outono); N, NNW, NE, SW e N (Inverno); N, NNE e SSW (Primavera), e N, NNW, SW e SSW (Verão). As predominâncias por períodos foram nas direções N, NW e SSW (Madrugada); N, NNW e NW (Manhã); N, NNE, S e SW (Tarde), e S, SW e SSW (Noite).
\end{abstract}

Palavras-chave: Baixada Fluminense, meteorologia de montanha, dispersão de poluentes, sistemas meteorológicos.

\section{Characterization of Wind Regime in Seropedica, Rio de Janeiro State, Brazil (2001-2010)}

\begin{abstract}
In this study, we characterize the surface wind regime in the municipality of Seropedica, Rio de Janeiro state, Brazil, based on 10 years of data on wind direction and speed collected by an Automated Surface Weather Station (ASWS). Histograms and wind roses were prepared decennially, seasonally, and by period (dawn, morning, afternoon and evening). The predominant decennial directions were $\mathrm{N}$ and SW; they were associated with the regimes of valley/mountain breeze and sea breeze along with the passage of Frontal Systems. The classes of decennial wind were calm (below $1 \mathrm{~m} . \mathrm{s}^{-1}$ ), $\left(40.2 \%\right.$ ) and light breeze (1 to $\left.3 \mathrm{~m} \cdot \mathrm{s}^{-1}\right),(39.8 \%)$. Seasonally, predominant directions were N, NW, NNE, SSW and SW (autumn); N, NNW, NE, SW and N (winter); N, NNE and SSW (spring); and N, NNW and SW, SSW (summer). By period, predominant directions were N, NW and SSW (dawn); N, NNW and NW (morning); N, NNE and S, SW (afternoon); and S, SW and SSW (evening).
\end{abstract}

Keywords: 'Fluminense' Lowlands, mountain meteorology, pollutant dispersion, meteorological systems. 


\section{INTRODUÇÃO}

O conhecimento do regime de vento é importante na caracterização da morfologia urbana, no conforto térmico de casas e edifícios, na dispersão de poluentes atmosféricos, nos estudos de qualidade do ar e na dinâmica dos focos de incêndios. Padrões de vento possuem uma grande variabilidade devido às influências de várias escalas, como, por exemplos, local (microescala), regional (mesoescala) e global (sinótica).

O clima de uma região pode ser resultado da persistência de certos tipos de circulações locais, principalmente nas regiões litorâneas e montanhosas, que se manifestam na forma de brisas marítima/ terrestre, vale/montanha e lacustre-baía (Stull, 2000; Whiteman, 2000). Essas circulações locais, quando afetadas por sistemas sinóticos, situação de alta pressão, sobressaem-se, enquanto na situação de baixa pressão, são sobrepostas por ventos sinóticos, os quais podem ser modificados pelas montanhas (Whiteman \& Doran, 1993; Latini et al., 2003). Nesse contexto, a topografia e os corpos d'água também afetam o regime de vento local (Oke, 1987).

A variação do vento depende de um número substancial de variáveis e parâmetros, que incluem: a radiação solar, a latitude, as interações mecânicas do terreno, a convecção e a força de Coriolis (Farrugia \& Micallef, 2006). Campo de vento varia espacialmente devido a latitude e longitude; na vertical, varia espacialmente na escala local, por causa dos efeitos geográficos; na escala regional, por causa dos efeitos sinóticos (Yamartino, 1984; Zängl et al., 2001).

A direção do vento é bastante variável no tempo e no espaço - principalmente quando os ventos são fracos -, em função da geografia do local, da rugosidade superficial $\left(\mathrm{z}_{0}\right)$, do relevo, da vegetação e da época do ano (Vendramini, 1986). Devido à grande importância do vento nas atividades humanas, o conhecimento de suas direções predominantes e velocidades médias se faz necessário no planejamento dessas atividades, seja em estudos e projetos de construção civil, produção agrícola e aproveitamento eólico para geração de energia (Sansigolo, 2005; Leite
\& Virgens Filho, 2006; Munhoz \& Garcia, 2008), seja na gestão da qualidade do ar.

O vento pode atuar como polinizador na agricultura e conhecer suas características é necessário na construção de quebra-ventos para proteção de projetos agrícolas. É utilizado como variável de entrada para cálculo de alguns modelos empíricos de evapotranspiração (ET) como, por exemplo, o método de Penman-Monteith, que pode determinar a necessidade, o consumo hídrico das culturas e o manejo de irrigação adequado àquela área e à determinada cultura (Munhoz \& Garcia, 2008).

Acompanhando toda a Região Metropolitana do Rio de Janeiro (RMRJ), no município de Seropédica e nas áreas circunvizinhas, tem ocorrido um elevado crescimento imobiliário e industrial nos últimos anos, e um novo aterro sanitário e uma rodovia em construção (Arco Metropolitano) são produtos desse crescimento. Mostra-se, assim, que caracterizar o regime do vento é de extrema importância para (o futuro) monitoramento e a avaliação do comportamento de poluentes gerados por essas atividades, assim como para o planejamento da morfologia urbana do município.

Foram realizados vários estudos sobre caracterização e importância do regime de vento na Cidade do Rio de Janeiro (CRJ) (Lyra et al., 2011; Zeri et al., 2011) e na RMRJ (Ferreira, 2005; Moraes et al., 2005; Rodrigues et al., 2007; Paiva, 2012). No entanto, há poucos estudos para a região de Seropédica sobre o comportamento dos ventos em superfície e altitude, destacando-se alguns estudos, como, por exemplos, Paiva (2012) e Jourdan (2007), que analisaram o regime dos ventos próximos à superfície para a RMRJ, incluindo o município de Seropédica por um período de cinco anos (20022006).

Baseado no exposto, o presente estudo tem por objetivo caracterizar o regime de vento em superfície na região de Seropédica, Rio de Janeiro, com base nos dados da Estação Meteorológica Automática de Superfície (EMAS), no período de 2001 a 2010 (dez anos), considerando a influência dos sistemas sinóticos, de mesoescala e local. 


\section{MATERIAL E MÉTODOS}

O município de Seropédica, situado a $22^{\circ} 44^{\prime}$ $38^{\prime \prime} \mathrm{S}$ de latitude, $43^{\circ} 42^{\prime} 27^{\prime \prime} \mathrm{W}$ de longitude e $26 \mathrm{~m}$ de altitude, no Estado do Rio de Janeiro (Figura 1), tem área de aproximadamente $266,55 \mathrm{~km}^{2}$. Embora considerado politicamente como integrante da RMRJ, geograficamente está situado em uma área da planície costeira fluminense denominada Baixada de Sepetiba. Limitada ao Sul pelo Oceano Atlântico, esta baixada possui como limites interiores a Serra do Mar a oeste (W) e noroeste (NW), o maciço da Pedra Branca a leste (E), a nordeste (NE) a Serra da Mantiqueira e, ao norte (N) e nordeste (NE), uma sucessão de morros de pequena altitude, que se incorporam à chamada paisagem de Mar de Morros (Jaccoud, 2006).

Foram coletados e organizados dados da direção $\left({ }^{\circ}\right)$ e da velocidade do vento $\left(\mathrm{m} . \mathrm{s}^{-1}\right)$ da EMAS de Seropédica, denominada de Ecologia Agrícola (EA), situada $22^{\circ} 45^{\prime} 28^{\prime \prime} \mathrm{S}$ de latitude, $43^{\circ} 41^{\prime} 5^{\prime \prime} \mathrm{W}$ de longitude e $34 \mathrm{~m}$ altitude, no período de 2001 a 2010. Os dados foram adquiridos no site do INMET (Instituto Nacional de Meteorologia) - (http:// www.inmet.gov.br/portal/index.php?r=estacoes/ estacoesAutomaticas). Por meio destes dados, foram elaborados histogramas e rosas dos ventos com o auxílio do programa WRPLOT (http://www. weblakes.com/products/wrplot/index.html).

A partir disso, foram feitas avaliações na escala decenal, sazonal e por períodos. Os períodos foram divididos da seguinte forma: madrugada (0-6h); manhã (6-12h); tarde (12-18h) e noite (18-23h). Após os dados serem organizados, foram feitas análise estatísticas descritivas (média, mediana, desvio padrão) e exploratórias (Box-plot).

Dados da Climanálise (http://www6.cptec.inpe. $\mathrm{br} /$ revclima/boletim/) foram usados na avaliação da frequência de atuação dos Sistemas Frontais (SF) no Estado do Rio de Janeiro (Climanálise, 2012) - Tabela 1. E, por fim, foram coletados artigos observacionais e numéricos sobre o regime de vento

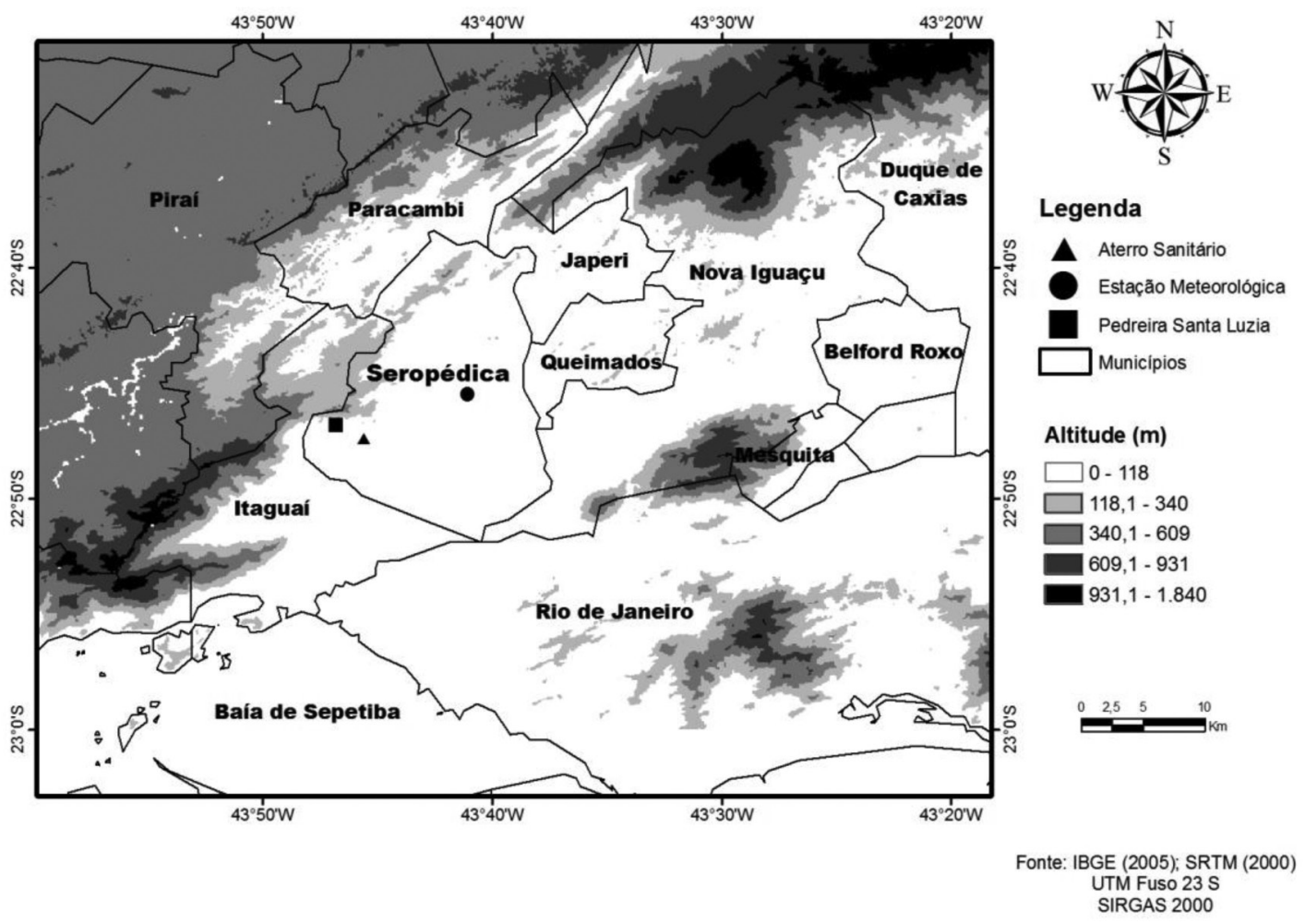

Figura 1. Município de Seropédica e sua área circunvizinha. Figure 1. Seropédica Municipality and its surrounding area. 
Tabela 1. Frequências Absoluta e Relativa (\%) dos Sistemas Frontais no Estado do Rio de Janeiro no período de 2001-2010.

Table 1. Absolute and Relative Frequency (\%) of Frontal Systems in the State of Rio de Janeiro in the period 2001-2010.

\begin{tabular}{lcc} 
Estações & $\begin{array}{c}\text { Frequência } \\
\text { Absoluta (FA) }\end{array}$ & $\begin{array}{c}\text { Frequência } \\
\text { Relativa (\%) (FR) }\end{array}$ \\
Verão & 61 & 18 \\
Outono & 78 & 23 \\
Inverno & 100 & 30 \\
Primavera & 99 & 29 \\
Total & 338 & 100 \\
\hline
\end{tabular}

Fonte: Climanalise - (http://www6.cptec.inpe.br/revclima/boletim/).

referente aos municípios e às localidades da zona oeste da RMRJ (Figura 1), para comparação dos resultados.

\section{RESULTADOS E DISCUSSÃO}

As direções predominantes identificadas na escala decenal no município de Seropédica foram Norte (N) e Sudoeste (SW), mas houve a ocorrência de outras direções, com menores frequências (Figura 2a). As direções SW e Sul-Sudoeste (SSW) correspondem à atuação dos sistemas de brisas (brisa marítima-terrestre) na região, principalmente da brisa da Baía de Sepetiba, juntamente com a passagem de SF na região, enquanto as direções $\mathrm{N}$ e Norte-Noroeste (NNW) correspondem à influência do regime de vento (brisa de vale-montanha) proveniente das cadeias montanhosas que circundam a região (Figura 1).

As maiores classes de velocidades do vento identificadas na escala decenal foram nas direções $\mathrm{Se}$ SW, ressaltando mais uma vez a atuação dos sistemas de mesoescala (circulação das brisas) e sinóticos (atuação dos SF), em particular nas estações de inverno e primavera (Tabela 1). Na distribuição das velocidades do vento decenal, verifica-se, claramente, a predominância do regime de calmarias (abaixo de $\left.1 \mathrm{~m} . \mathrm{s}^{-1}\right), 40,2 \%$ na região de Seropédica, seguidas de uma segunda classe de ventos entre 1 e $3 \mathrm{~m} \cdot \mathrm{s}^{-1}$ (ventos fracos), $39,8 \%$, segundo o padrão de medidas de vento em superfície da Organização Mundial de Meteorologia (OMM) - (WMO, 2000).
Esse tipo de classe implica em dificuldades na dispersão de poluentes, principalmente de $\mathrm{MP}_{10}$, visto que a região atualmente comporta um aterro sanitário, seguido de um aumento considerável do tráfego de caminhões e carros na Estrada Rio-São Paulo (Rodovia BR-465). As demais classes ficaram entre 2 e $4 \mathrm{~m} \cdot \mathrm{s}^{-1}(19,5 \%)$ - brisa suave (WMO, 2000) - e com pouca influência de classe de ventos entre 4 a $6 \mathrm{~m} \cdot \mathrm{s}^{-1}$, somando apenas $0,5 \%$ (Figura $2 \mathrm{~b}$ ).

Através do box-plot, mostrou-se que as velocidades médias mensais ficaram abaixo de $6 \mathrm{~m} . \mathrm{s}^{-1}$ registrados em toda a série de dez anos, sendo o mês de março com menor valor da velocidade média na escala decenal, da ordem de $2,8 \mathrm{~m} \cdot \mathrm{s}^{-1}$. Na análise das velocidades médias mensais do vento em Seropédica, revelaram-se valores acima de 2,9 $\mathrm{m} . \mathrm{s}^{-1}$. Ou seja: o regime médio da velocidade do vento na região de Seropédica não se modifica, em virtude da área de estudo estar em fundo de vale, o que representa dificuldade em dispersar os poluentes e, como consequência, é uma influência negativa na qualidade do ar da região, em função do aumento de empreendimentos imobiliários e industriais nos próximos anos.

Sazonalmente, ocorre uma variação das velocidades médias, entre 2,8 e $8,7 \mathrm{~m} \cdot \mathrm{s}^{-1}$ (vento fraco à brisa moderada, WMO, 2000) - (Figura 3), principalmente nas estações de transição (outono e primavera). Isso se deve à ocorrência do regime de brisas de vale/montanha acoplado ao regime de brisa marítima/terrestre, juntamente com a entrada de SF nestas estações (Tabela 1).

$\mathrm{Na}$ escala mensal, o regime médio da velocidade do vento se modificou nos meses de fevereiro, maio, julho, outubro, novembro e dezembro, mostrandose superior a $7 \mathrm{~m} \cdot \mathrm{s}^{-1}$, em virtude de a região de estudo sofrer influência de várias forçantes (cadeia montanhosa e da Baía de Sepetiba) e dos sistemas meteorológicos de várias escalas, que alteram o padrão de intensidade do vento.

Foram identificadas as seguintes direções predominantes: NW,NNE- quadrante N, e as direções SW e $S$ - quadrante $S$, para ambas as estações do ano (Figuras 4 e 5b), respectivamente, que seguiram um padrão similar às direções identificadas na escala decenal. No verão (Figura 4a), as predominâncias foram nas direções SW, SSW e N, com maiores 


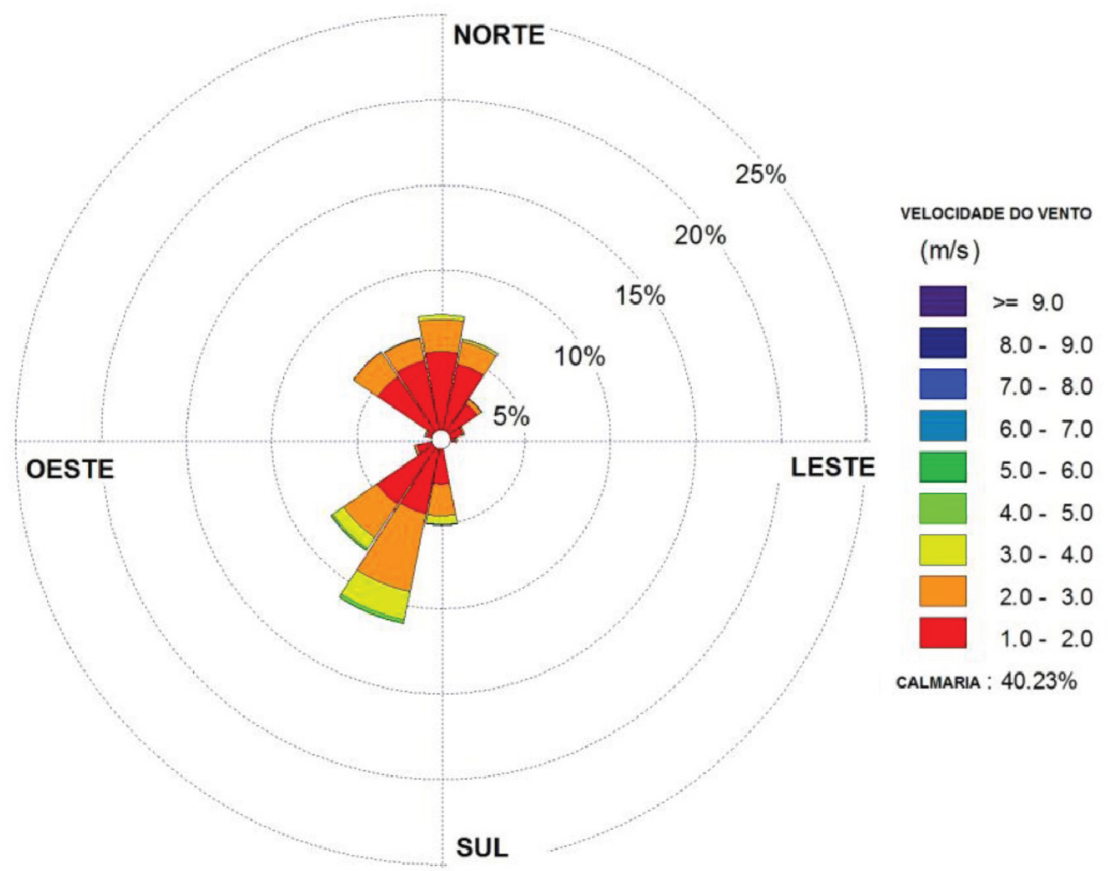

(a)

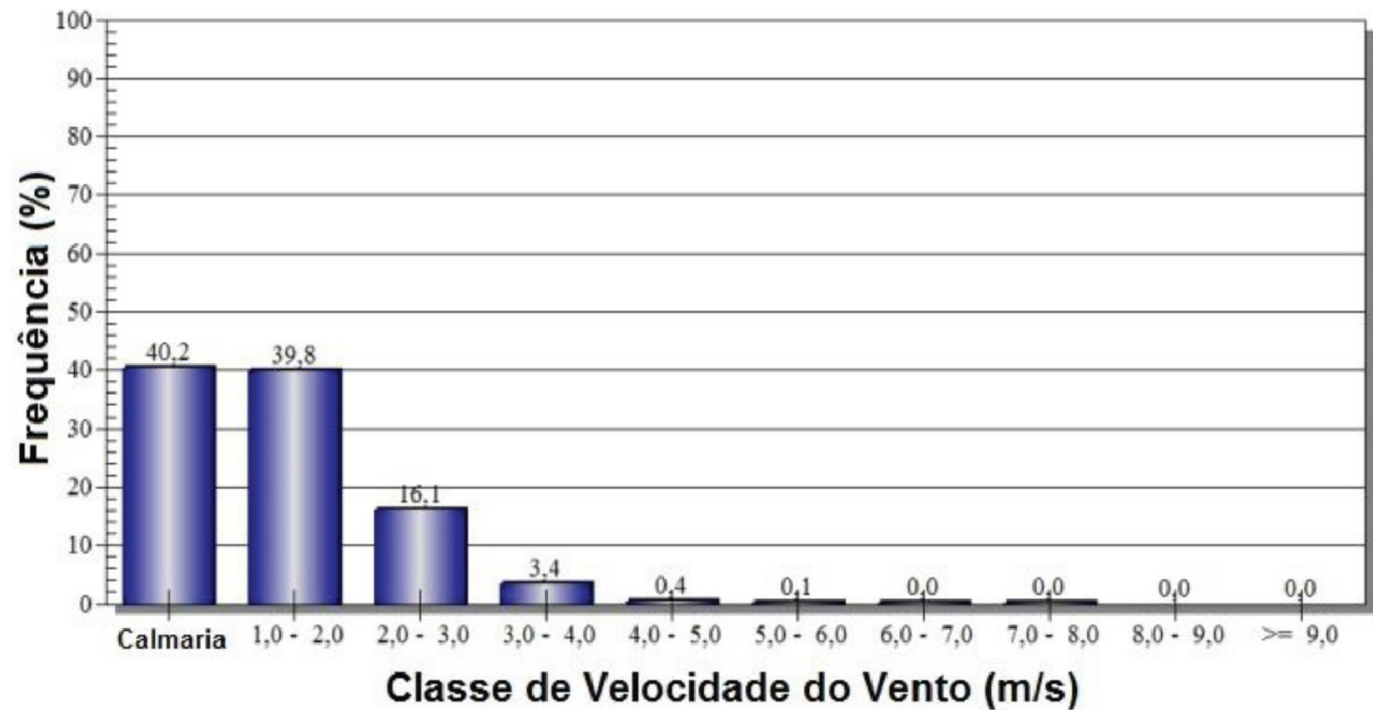

Figura 2. Rosa dos ventos (a) e histograma das velocidades dos ventos decenal (b) do município de Seropédica (2001-2010).

Figure 2. Wind rose (a) and wind speeds decennial histogram (b) of Seropédica Municipality (2001-2010).

velocidades principalmente no quadrante $S$, correspondente à atuação dos SF que, nesta época do ano, têm sua trajetória lenta e organizam maior convecção no interior do continente (Ferreira, 2005; Moraes et al., 2005; Rodrigues et al., 2007), apesar de ser a estação com menor frequência (18\%) de SF no Estado do Rio de Janeiro.
$\mathrm{Na}$ estação da primavera (Figura 5b), os regimes predominantes foram nas direções de S a SW (brisas marítimas), concordando com os estudos realizados anteriormente na região (Jourdan, 2007). A estação do outono caracterizou-se por regime de ventos fracos do quadrante $\mathrm{N}$ e maiores velocidades no quadrante $S$, sendo que nesta estação foram encontradas 


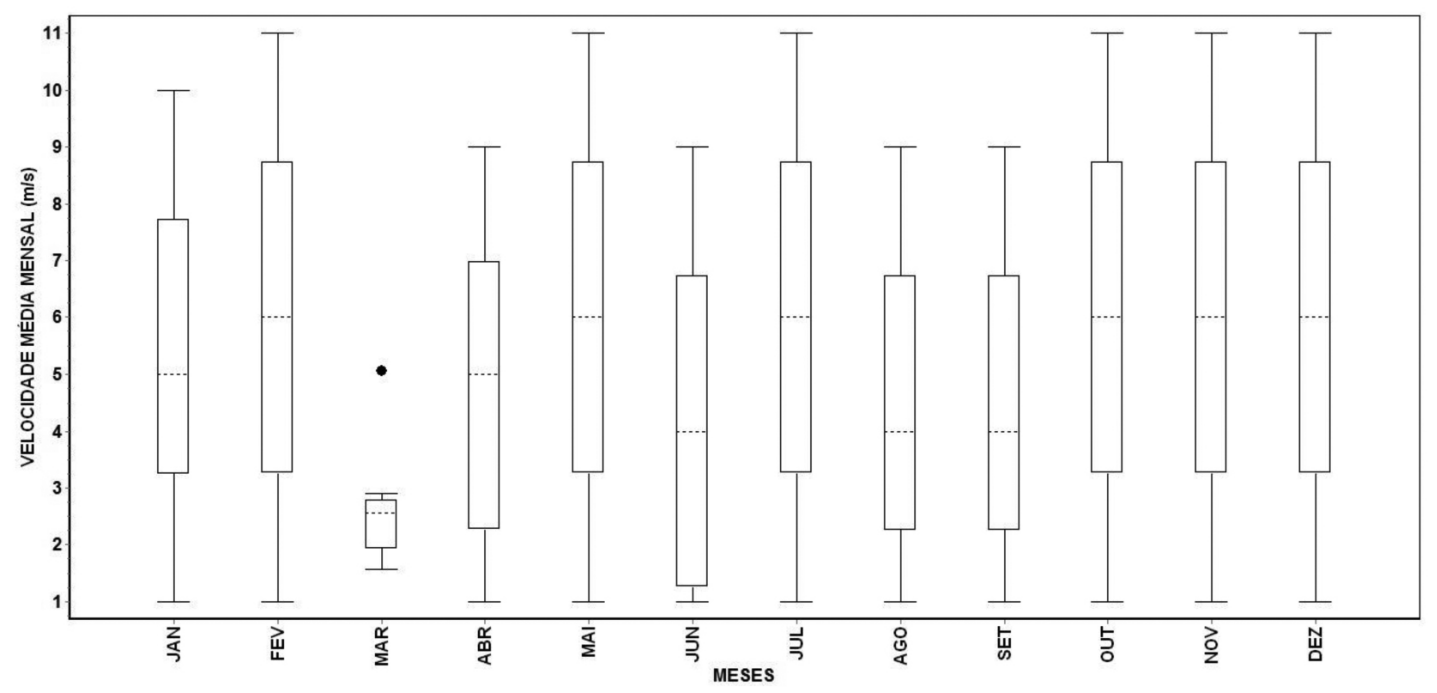

Figura 3. Box-plot da velocidade média mensal do Município de Seropédica (2001-2010). Figure 3. Box Plot Monthly average Speed on Seropédica Municipality (2001-2010).
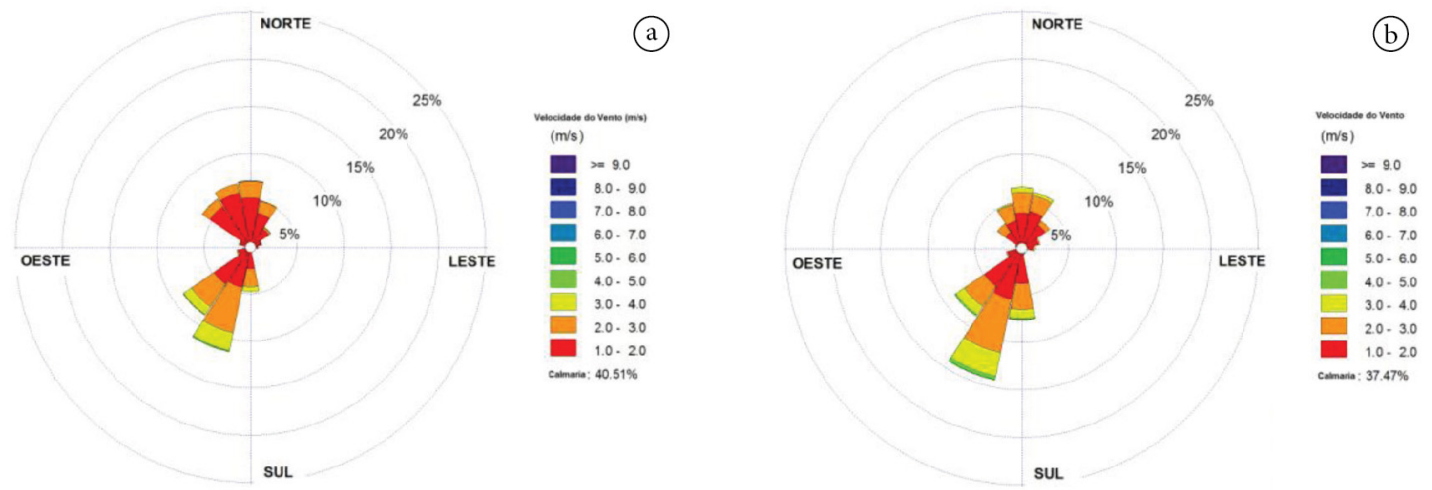

Figura 4. Rosa dos ventos da estação de verão (a) e da estação de primavera (b) do município de Seropédica (20012010).

Figure 4. Wind Rose on summer season (a) and spring season (b) on Seropédica Municipality (2001-2010).

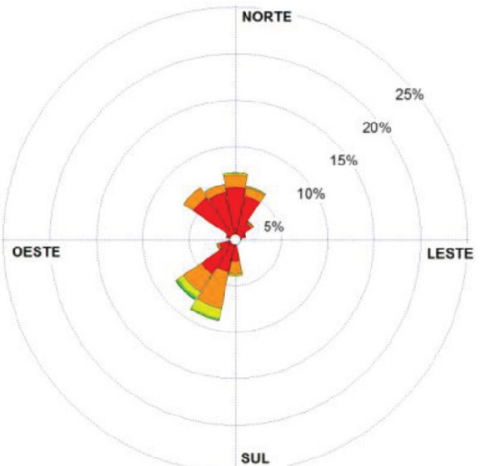

(a)

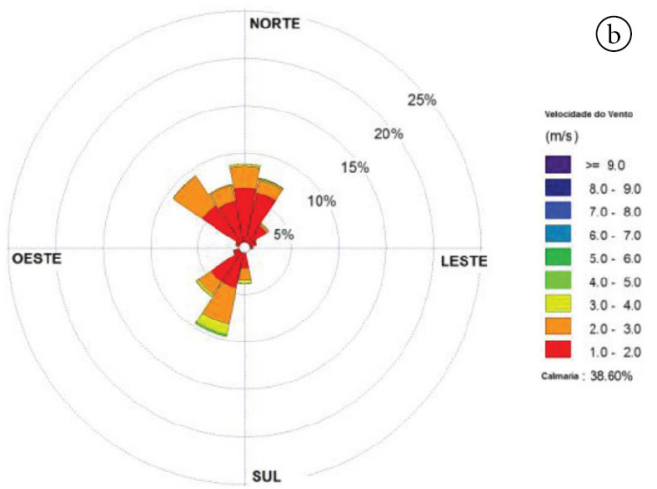

Figura 5. Rosa dos ventos da estação de outono (a) e da estação de inverno (b) do município de Seropédica (20012010).

Figure 5. Wind Rose on autumn season (a) and winter season (b) on Seropédica Municipality (2001-2010). 
as maiores velocidades do vento na direção SW, correspondente à atuação dos SF (Figura 5a). No inverno (Figura $5 \mathrm{~b}$ ), o quadrante $\mathrm{S}$ mantém suas maiores velocidades nas direções $\mathrm{S}$ e SW; entretanto, o quadrante $\mathrm{N}$ ( $\mathrm{N}$ e NNE) começa aumentar a sua frequência na região, devido à atuação da Alta Subtropical do Atlântico Sul (ASAS), conforme identificado nos estudos de Dereczynski et al. (2009), Lyra et al. (2011) e Zeri et al. (2011).

$\mathrm{Na}$ Figura 6, notou-se um percentual elevado da classe de calmaria no município de Seropédica, independentemente da sazonalidade, durante todo o período de estudo. Na estação de verão, a classe de calmaria foi similar à estação da primavera (Figura 6a). Nota-se que, na estação de outono, foi identificado um percentual de 52,4\% de calmaria, enquanto o restante do percentual foi redistribuído para as demais classes (Figura 6b).

No inverno, o percentual da classe de calmaria decresce em 12\% em comparação ao outono (Figura 6c). Esse elevado número percentual da calmaria é prejudicial ao transporte e à dispersão dos poluentes na região, que poderá a ter implantação de indústrias nos próximos anos.

Para a estação da primavera (Figura 6d), há maior influência da classe de ventos de 3 a $5 \mathrm{~m} \cdot \mathrm{s}^{-1}$ (brisa suave - WMO, 2000), totalizando 5\%. Porém, a classe de ventos que não ultrapassam $1 \mathrm{~m} \cdot \mathrm{s}^{-1}$ aumenta consideravelmente, com valor percentual de $44 \%$. Nas Figuras 6a, 6b e 6d, observa-se que a classe de vento de 1 a $3 \mathrm{~m} \cdot \mathrm{s}^{-1}$ (ventos fracos - WMO, 2000), juntamente com a situação de calmarias, foi a predominante na escala decenal. Em suma, as maiores velocidades encontradas no estudo concentram-se apenas na estação da primavera e os menores valores, na estação de outono.

O período da madrugada (Figura 7a) foi caracterizado pelas predominâncias nas direções $\mathrm{N}$ e NW, seguidas de menores ocorrências nas direções SSW e SW. Durante o período de manhã (Figura 7b), ocorre um aumento na frequência na direção NW, seguido de uma diminuição significativa nas direções SSW e SW. À tarde (Figura 8a), ocorreu uma redistribuição na direção do vento e um aumento significativo da direção SW, seguida da direção N. No período da noite (Figura $8 \mathrm{~b}$ ), os ventos de quadrante $\mathrm{N}$ diminuem significativamente, enquanto os ventos de quadrante $S$ aumentam consideravelmente, principalmente nas direções SSW e SW.

No período madrugada (Figura 9a), notase um total elevado da classe de calmaria, com $62,6 \%$, seguido da classe de ventos fracos - 32,6\%
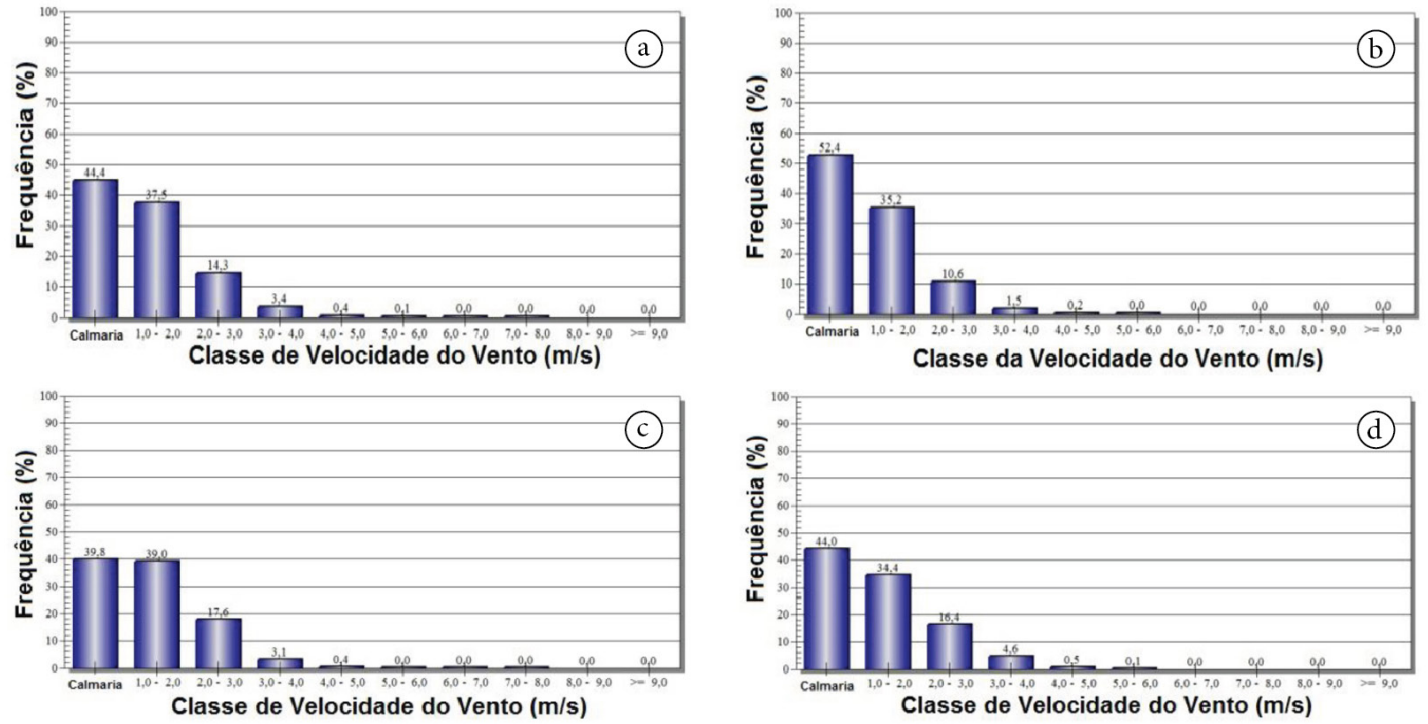

Figura 6. Histograma das velocidades dos ventos nas estações de verão (a) outono, (b), inverno (c) e primavera (d) do município de Seropédica (2001-2010).

Figure 6. Wind speeds Histogram during summer (a), autumn (b), winter (c) and spring (d) on Seropédica Municipality (2001-2010). 


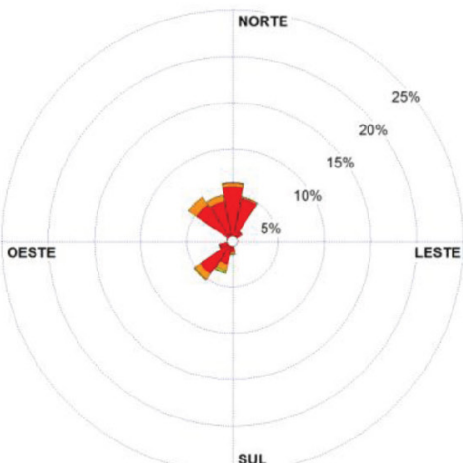

(a)

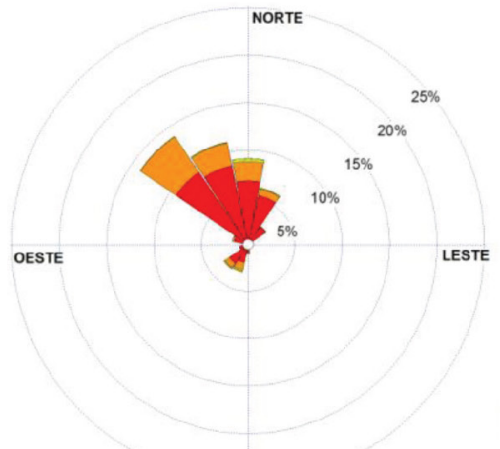

(b)

sul

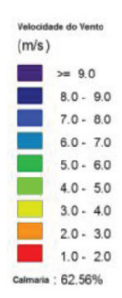

sul

Figura 7. Rosa dos ventos do período da madrugada (a) e do período da manhã (b) do município de Seropédica (2001-2010).

Figure 7. Wind Rose on before sunrise (a) and morning (b) on Seropédica Municipality (2001-2010).
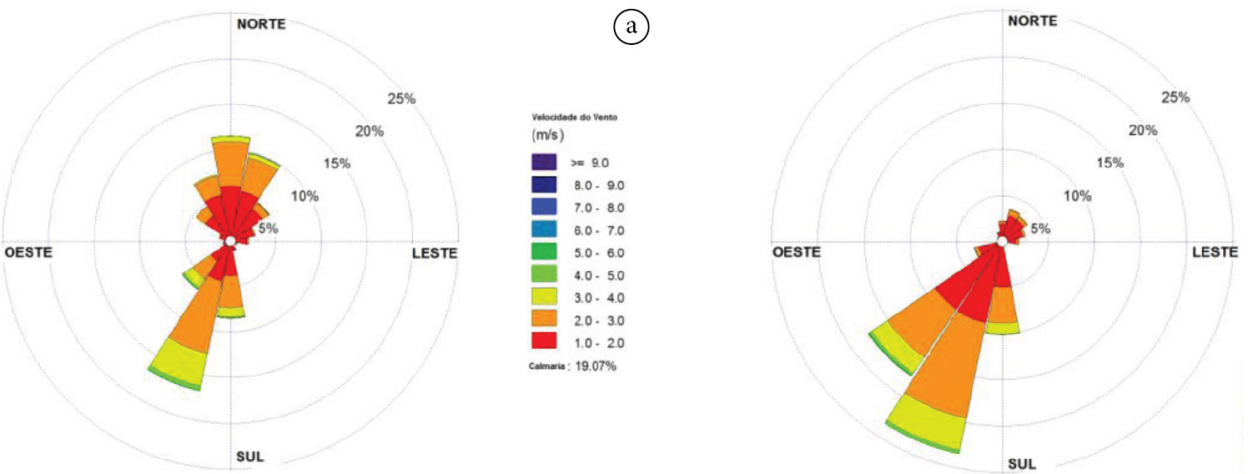

(b)

Figura 8. Rosa dos ventos do período da tarde (a) e do período da noite (b) do município de Seropédica (2001-2010).

Figure 8. Wind Rose on afternoon (a) and twilight (b) on Seropédica Municipality (2001-2010).
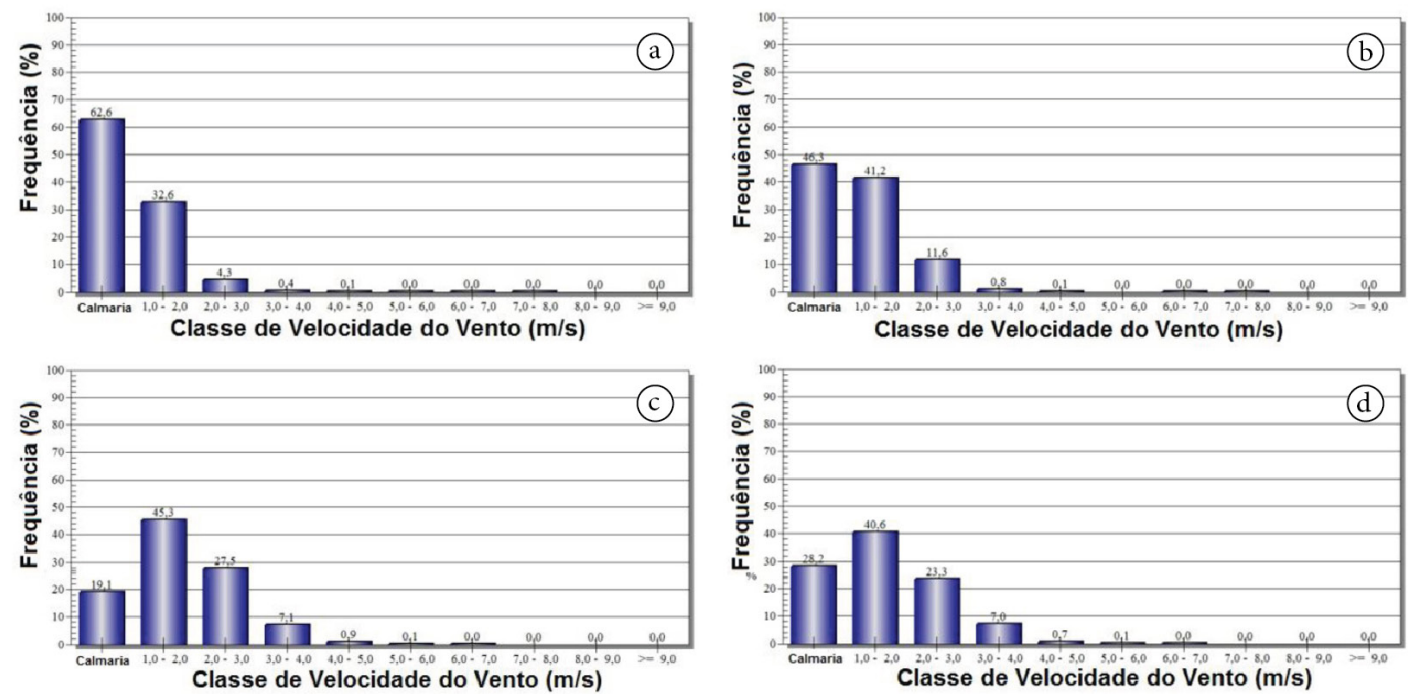

Figura 9. Histograma das velocidades dos ventos no período da madrugada (a), manhã (b), tarde (c) e noite (d) do município de Seropédica (2001-2010).

Figure 9. Wind speeds Histogram during dawn (a) morning (b), afternoon (c) night and (d) on Seropédica Municipality (2001-2010). 
(1-3 m.s $\left.\mathrm{s}^{-1}\right)$ - (WMO, 2000). Diversamente, no período da manhã (Figura 9b), a intensidade dos ventos fracos aumenta significativamente, com a marca de $53 \%$, e ainda persiste uma elevada situação de calmaria, mas com um menor percentual em comparação ao período da madrugada. No período da tarde (Figura 8c), a situação de calmaria tende a uma redução significativa em comparação aos períodos anteriores, 19,1\%, seguida de um aumento da classe de ventos fracos até classe moderada, em virtude da atuação da circulação da brisa marítima. À noite (Figura 9d), o regime de ventos fracos ainda persiste com percentual de $40,6 \%$ do total registrado, assim como a classe de ventos moderados, em função da atuação da brisa terrestre na região.

$\mathrm{Na}$ análise da frequência das direções do vento no ciclo diário-sazonal, a partir das direções cardeais e colaterais na escala decenal (Figura 10), foram identificadas, no verão, as maiores frequências na direção SW, entre às $17 \mathrm{~h}(25 \%)$ e $23 \mathrm{~h}(28 \%)$, principalmente às $21 \mathrm{~h}(42 \%)$, seguida da direção $\mathrm{S}$, entre às $16 \mathrm{~h}(26 \%)$ e $20 \mathrm{~h}(25 \%)$, da direção $\mathrm{N}$, entre os horários $11 \mathrm{~h}$ (24\%) e $15 \mathrm{~h}$ (25\%), e por fim na direção NW, entre às 7 h (23\%) e 10h (24\%). Vale ressaltar que houve um aumento da direção NE ocorrido nesta estação devido à atuação do ASAS (Figura 10a).

As maiores frequências identificadas no outono foram na direção SW entre às $18 \mathrm{~h}(27 \%)$ e $22 \mathrm{~h}$ (34\%), em particular às $21 \mathrm{~h}(42 \%)$, similar à estação de verão, seguida da direção S entre às $16 \mathrm{~h}$ (23\%) e $20 \mathrm{~h}$ (25\%), da direção $\mathrm{N}$ entre às $12 \mathrm{~h}$ (28\%) e 15h (26\%), sendo que o período difere do verão, com diferença de uma hora. Por fim, na direção NW entre às $9 \mathrm{~h}$ (26\%) e 10h (24\%), em comparação ao verão, há uma diferença no horário de ocorrência de duas horas. A diferença no horário entre as estações consiste na quantidade de radiação solar recebida pelas cadeias montanhosas na região de estudo.

No inverno, as frequências na direção SW foram às $19 \mathrm{~h}(27 \%), 21 \mathrm{~h}(33 \%)$ e $22 \mathrm{~h}(34 \%)$, similar ao verão e ao outono; no entanto, ocorreu uma diminuição significativa na frequência desta direção, seguida da direção $S$ entre às $17 \mathrm{~h}$ (22\%) e 20h (23\%), da direção N entre 12h (27\%) e 16h (26\%), verificandose um aumento em mais uma hora em comparação à estação anterior, pois o período é diferente em comparação às estações anteriores; e, por fim, a direção NW entre às 9h (26\%) e 10h (24\%), similar à estação anterior e diferindo, significativamente, da estação de verão. O aumento da direção NE, nesta estação, refere-se à migração do ASAS, que favorece o aumento desta direção.

Novamente na primavera, as maiores frequências identificadas foram na direção SW entre às $18 \mathrm{~h}(27 \%)$ e $23 \mathrm{~h}(22 \%)$, em particular às $21 \mathrm{~h}(42 \%)$, similar às estações de verão e outono. A direção $S$ entre às $17 \mathrm{~h}$ (27\%) e 20h (25\%), a direção N entre os horários $12 \mathrm{~h}$ (28\%)e 16h (26\%), similar à estação anterior e, por fim, na direção NW, entre 8h (23\%) e 12h (24\%), em comparação às estações anteriores, há uma diferença no horário de ocorrência de duas horas.

As principais direções identificadas na escala sazonal-horária (decenal) foram às direções N e NW, e $\mathrm{S}$ e SW, correspondentes à atuação das brisas de vale/montanha, brisa marítima/terrestres e os SFs. A direção NW é tipicamente da ocorrência da brisa de vale. Durante o dia, a cadeia montanhosa que circunda a região de Seropédica fica exposta à maior quantidade de radiação solar e, portanto, aquece o ar. Desta forma, desenvolve-se uma gradiente de pressão, que faz com que o ar movimente-se vale acima, gerando brisa de vale.

A direção $\mathrm{N}$ consiste na interação das montanhas e do vale existente na região de estudo, que ajudou a canalizar a brisa, bem como favorece sua formação. No período da madrugada até início da manhã, há atuação conjunta da brisa de montanha e da brisa terrestre, após os horários entre $12 \mathrm{~h}$ e $16 \mathrm{~h}$, sendo que novamente há um acoplamento desse regime de vento com a brisa marítima. Vale ressaltar que a direção $\mathrm{N}$ persiste ao longo do ciclo diário e sazonalmente, dando a entender que ocorre um efeito de canalização do vento a partir do acoplamento dos regimes citados anteriormente.

As direções $S$ e SW referem-se à atuação da circulação da brisa, seguida da ocorrência de sistemas frontais na região (SW). A maior frequência da direção SW nas estações de outono, inverno e primavera (Tabela 1) se deve à atuação de SFs no Estado do Rio de Janeiro (Ferreira, 2005; Dereczynski et al., 2009).

A Tabela 2 faz referência aos estudos envolvendo a caracterização do regime de ventos na RMRJ. De 

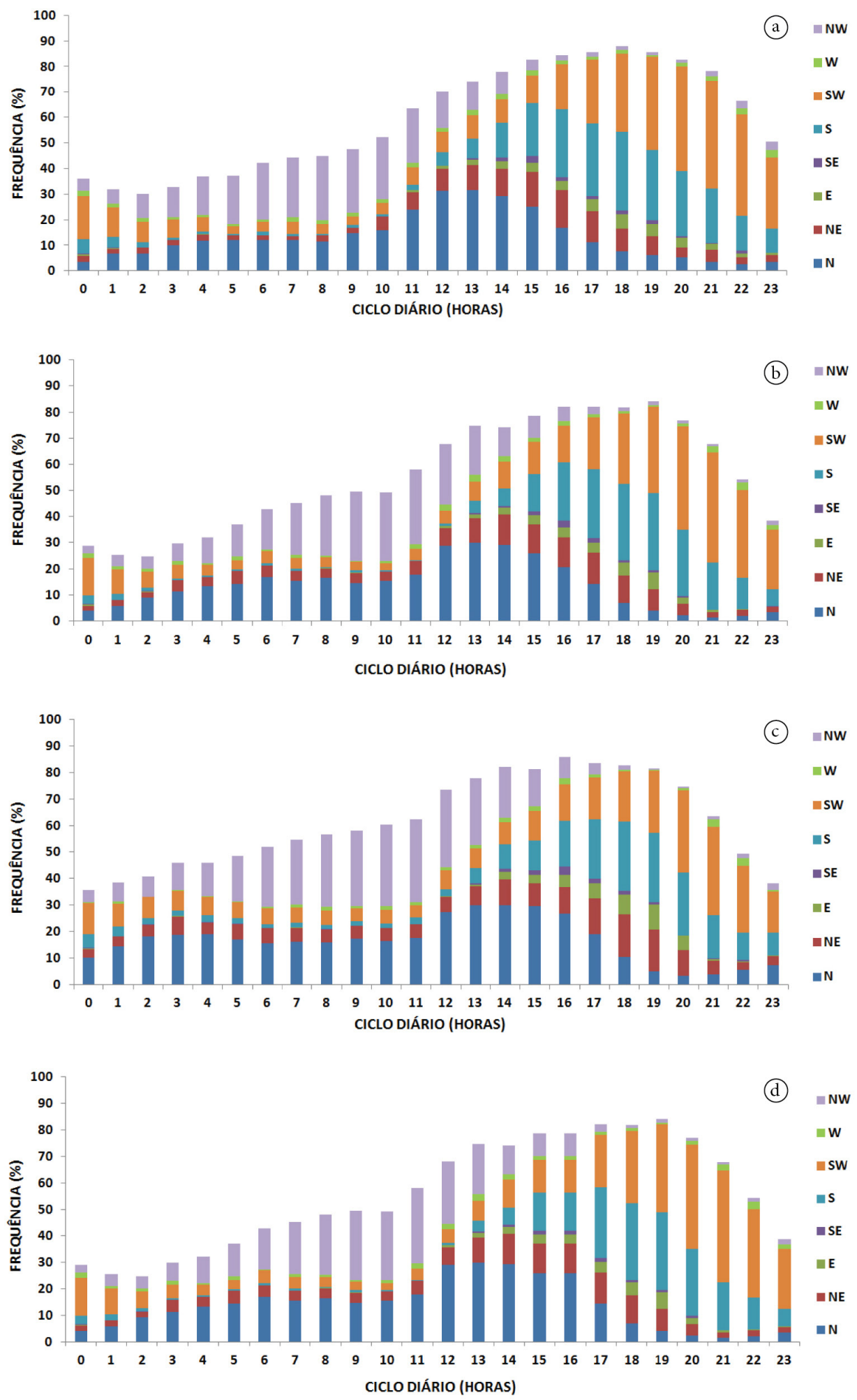

Figura 10. Frequência (\%) horária da direção do vento (giro) nas estações de verão (a), outono (b), inverno (c) e primavera (d) no período de 2001-2010.

Figure 10. Hourly wind direction (rotation) Frequency (\%) on summer (a), autumn (b) winter (c) and spring (d) seasons in 2001-2010 period. 
Tabela 2. Estudos sobre regime de vento na Região Metropolitana do Rio de Janeiro.

Table 2. Wind regime study on Rio de Janeiro Metropolitan Region.

\begin{tabular}{|c|c|c|c|c|}
\hline Título & Autores & $\begin{array}{l}\text { Sistemas Meteo- } \\
\text { rológicos }\end{array}$ & Localidade & $\begin{array}{c}\text { Direções } \\
\text { Predominantes } \\
\text { (Cardeal) }\end{array}$ \\
\hline $\begin{array}{l}\text { Caracterização do Regime de } \\
\text { Vento Próximo À Superfície } \\
\text { na Região Metropolitana do } \\
\text { Rio de Janeiro no Período } \\
\text { 2002-2006 }\end{array}$ & Jourdan (2007) & $\begin{array}{l}\text { Brisa Marítima/ } \\
\text { Terrestre }\end{array}$ & Seropédica - RJ & $\begin{array}{l}\text { SW a S - tarde } \\
\text { e noite/ NW a } \\
\text { NNE - } \\
\text { madrugada e } \\
\text { manhã }\end{array}$ \\
\hline $\begin{array}{l}\text { Climatologia da Precipitação } \\
\text { no Município do Rio de Janeiro }\end{array}$ & $\begin{array}{l}\text { Dereczynski et al. } \\
\qquad(2009)\end{array}$ & $\begin{array}{c}\text { ASAS; Brisas Marítima } \\
\text { /Terrestre }\end{array}$ & Rio de Janeiro - RJ & $\begin{array}{l}\text { Nordeste; } \\
\text { SW - tarde } \\
\text { e noite/ } \\
\text { NW - madru- } \\
\text { gada e manhã }\end{array}$ \\
\hline $\begin{array}{l}\text { Spatiotemporal analysis of } \\
\text { particulate matter, sulfur } \\
\text { dioxide and carbon monoxide } \\
\text { concentrations over the city of } \\
\text { Rio de Janeiro, Brazil }\end{array}$ & Zeri et al. (2011) & $\begin{array}{l}\text { Sistemas Frontais, Bri- } \\
\text { sas Marítima/Terrestre }\end{array}$ & Rio de Janeiro - RJ & $\begin{array}{l}\text { ESE, NW, W e } \\
\text { WSW }\end{array}$ \\
\hline $\begin{array}{l}\text { Aporte atmosférico de amônio, } \\
\text { nitrato e sulfato em área de } \\
\text { floresta ombrófila densa montana } \\
\text { na Serra dos Órgãos, RJ }\end{array}$ & $\begin{array}{l}\text { Rodrigues et al. } \\
\qquad(2007)\end{array}$ & $\begin{array}{l}\text { Brisas Marítima/ } \\
\text { Terrestre }\end{array}$ & $\begin{array}{l}\text { Rio de Janeiro - RJ } \\
\text { (Baía de Guanabara) }\end{array}$ & $\mathrm{NeS}$ \\
\hline $\begin{array}{l}\text { Modelagem em Alta Resolução } \\
\text { do Escoamento Atmosférico } \\
\text { Usando o ARPS na Região } \\
\text { Metropolitana do Rio de } \\
\text { Janeiro }\end{array}$ & Paiva (2012) & $\begin{array}{l}\text { Brisas Marítima/ } \\
\text { Terrestre }\end{array}$ & $\begin{array}{l}\text { Seropédica/Itaguaí } \\
\text { (Baía de Sepetiba) }\end{array}$ & SW, E e SE \\
\hline
\end{tabular}

Fontes: SBMET - Sociedade Brasileira de Meteorologia (2012). Revista Brasileira de Meteorologia (RBMET), disponível no seguinte endereço eletrônico: http://www.sbmet.org.br/portal2011/index.php, e NCQAr - Núcleo Computacional de Estudo da Qualidade do Ar (2012). Produção Científica, disponível no seguinte endereço eletrônico: https://sites.google.com/a/lamma.ufrj.br/ncqar-beta/ producao-cientifica.

acordo com a literatura, as brisas marítima/terrestre são os regimes predominantes na circulação local e se mantêm ao longo do ano, apesar da atuação de sistemas meteorológicos da escala sinótica e dos efeitos locais (topografia e Baía de Sepetiba). Os resultados encontrados neste estudo corroboram com os resultados encontrados por Jourdan (2007), Dereczynski et al. (2009) e Paiva (2012).

\section{CONCLUSÕES}

O município de Seropédica-RJ, na avaliação decenal, possui como direções predominantes o N (brisa de vale/montanha) e SW (brisa marítima - sistema frontal).

Os regimes da velocidade do vento identificados em Seropédica são calmaria e ventos fracos. Em suma, as maiores velocidades encontradas no estudo são concentradas nas estações primavera, inverno e verão, e os menores valores, na estação do outono.

As velocidades médias mensais do vento em Seropédica na escala decenal são caracterizadas como brisa suave. Na escala sazonal, a velocidade média do vento varia entre vento fraco e brisa moderada nas estações outono e primavera, em virtude da ocorrência do regime de brisas de vale/ montanha acoplado ao regime de brisa marítima/ terrestre, juntamente com entrada de sistemas frontais na região. $\mathrm{Na}$ escala mensal, o regime médio do vento é modificado nos meses de fevereiro, maio, julho, outubro, novembro e dezembro em razão da influência de várias forçantes (cadeia montanhosa e da Baía de Sepetiba) e dos sistemas meteorológicos de várias escalas na região.

$\mathrm{Na}$ avaliação sazonal e por períodos, as direções predominantes são no quadrante $\mathrm{N}$ e S. As direções no quadrante $\mathrm{N}$ e $\mathrm{S}$ são devidas à circulação das 
brisas de montanha/vale, visto que a região de Seropédica é circundada por uma série de cadeias montanhosas. Note-se que a permanência constante do regime de ventos nas direções NE e NNE deve-se à atuação do ASAS. Sazonalmente, o regime de vento no município de Seropédica segue o mesmo padrão da escala decenal.

O estudo revela que o padrão de ventos pouco intensos na região deve ser levado em consideração em futuras concessões ambientais para instalações de empreendimentos poluidores, uma vez que os avanços econômicos esperados com os novos empreendimentos podem não ser tão vantajosos, já que potenciais aumentos de gastos com saúde pública equilibrariam a balança econômica, sem contarmos os riscos à saúde da população.

Por fim, as principais recomendações são o aumento do número de estações meteorológicas automáticas na localidade de Seropédica e nas áreas circunvizinhas, e de estudos numéricos sobre o escoamento local.

\section{AGRADECIMENTOS}

Os autores agradecem ao INMET, pela disponibilidade dos dados meteorológicos da EMAS de Seropédica, no período de 2001-2010. Os sinceros agradecimentos ao CPTEC/INPE, pelo fornecimento dos dados do boletim da CLIMANÁLISE. Os autores agradecem à Carolina Gomes Moreira, pelo seu trabalho de Especialização realizado em Seropédica, sendo ponto de partida para a série de trabalhos sobre a região.

\section{STATUS DA SUBMISSÃO}

Recebido: 02/10/2012

Aceito: 07/10/2013

Publicado: 31/12/2013

\section{AUTOR(ES) PARA CORRESPONDÊNCIA}

\section{José Francisco Oliveira Júnior}

Departamento de Ciências Ambientais - DCA, Universidade Federal Rural do Rio de Janeiro - UFRRJ, CEP 23890-000, Seropédica, RJ, Brasil e-mail: jfoliverjr@lamma.ufrj.br

\section{REFERÊNCIAS}

Climanálise. Produtos Climanálise INPE/CPTEC. 2012. [cited 2012 jul. 31]. Available from: www.cptec.inpe.br/ products/climanalise/.

Dereczynski CP, Oliveira JS, Machado CO. Climatologia da Precipitação no Município do Rio de Janeiro. Revista Brasileira de Meteorologia 2009; 24(1): 24-38. http:// dx.doi.org/10.1590/S0102-77862009000100003

Farrugia PS, Micallef A. Comparative analysis of estimators for wind direction standard deviation. Meteorological Applications 2006; 13(1): 29-41. http:// dx.doi.org/10.1017/S1350482705001982

Ferreira MS. Simulação do Transporte de Poluentes Atmosféricos na Bacia Aérea III da Região Metropolitana do Rio de Janeiro Via o Modelo AERMOD [dissertação]. Rio de Janeiro: Universidade Federal do Rio de Janeiro; 2005.

Instituto Brasileiro de Geografia e Estatística - IBGE. Brasil em Relevo (Malha). IBGE, 2000. [cited 2005 out]. Available from: http://www.ibge.gov.br.

Jaccoud CFS. Produção de Biomassa de Eucalyptus Urophylla em Três Classes de Solos em Seropédica, RJ [monografia]. Seropédica: Universidade Federal Rural do Rio de Janeiro; 2006.

Jourdan P. Caracterização do Regime de Ventos Próximo à Superfície na Região Metropolitana do Rio de Janeiro [monografia]. Rio de Janeiro: Universidade Federal do Rio de Janeiro; 2007.

Latini G, Grifoni RC, Passerini TGS. Modelling the Interactions Between Sea Breezes and Valley Breezes. In: Anais 12th Conference on Interactions of the Sea and Atmosphere; 2003; Ancona. America Meteorological Society; 2003.

Leite ML, Virgens Filho JS. Avaliação da velocidade média e direção predominante do vento em Ponta Grossa-PR. Revista Brasileira de Agrometeorologia 2006; 14(2): 157-167.

Lyra GB, Oda-Souza M, Viola DN. Modelos Lineares Aplicados À Estimativa da Concentração do Material Particulado $\left(\mathrm{PM}_{10}\right)$ na Cidade do Rio de Janeiro, RJ. Revista Brasileira de Meteorologia 2011; 26(3): 392-400. http://dx.doi.org/10.1590/S0102-77862011000300006

Moraes NO, Marton E, Pimentel, LCG. Simulações Numéricas da Formação de Ilha de Calor na Região Metropolitana do Rio de Janeiro. Anuário do Instituto de Geociências (Rio de Janeiro)2005; 28(2): 116-138.

Munhoz FC, Garcia A. Caracterização da Velocidade e Direção Predominante dos Ventos para a Localidade de Ituverava-SP. Revista Brasileira de Meteorologia 2008; 23(1): 30-34. http://dx.doi.org/10.1590/S010277862008000100003 
Núcleo Computacional de Estudo da Qualidade do Ar - NCQAr. Produção Científica; 2013. [cited 2012 jul. 1] Available from: https://sites.google.com/a/lamma. ufrj.br/ncqar-beta/producao-cientifica.

Oke TR. Boundary Layer Climates. 2nd ed. Cambridge: University Press; 1987.

Paiva LMS. Modelagem em Alta Resolução do Escoamento AtmosféricoUsando o ARPS na Região Metropolitana do Rio de Janeiro [tese]. Rio de Janeiro: Universidade Federal do Rio de Janeiro; 2012.

Rodrigues RAR, Mello WZ, Souza AP. Aporte atmosférico de amônio, nitrato e sulfato em área de floresta ombrófila densa montana na Serra dos Órgãos, RJ. Quimíca Nova 2007; 30(8): 1842-1848. http://dx.doi. org/10.1590/S0100-40422007000800009

Sansigolo C. Distribuições de Probabilidade de Velocidade e Potência do Vento. Revista Brasileira de Meteorologia 2005; 20(2): 207-214.

Shuttle Radar Topography Mission - SRTM. NASA Jet Propulsion Laboratory. The objective of the Shuttle Radar Topography Mission is to obtain elevation radar data on a near-global scale to generate the most complete high-resolution digital topographic database of Earth. The SRTM radar data have many applications. [cited 2000 maio]. Available from: http://www2.jpl.nasa.gov/ srtm/missionoverview.html.

Sociedade Brasileira de Meteorologia - SBMET. Revista Brasileira de Meteorologia (RBMET). 2012. [cited 2012 jul. 31]. Available from: http://www.sbmet.org.br/ portal2011/index.php/.

Stull RB. Meteorology for Scientists and Engineers. Massachusetts: Brooks/Cole Pacific Grove; 2000.

Vendramini EZ. Distribuições probabilísticas de velocidades do vento para avaliação do potencial energético eólico [tese]. Botucatu: Universidade Estadual Paulista; 1986.

Whiteman CD, Doran JC. The Relationship between Overlying Synoptic-Scale Flows and Winds within a Valley. Journal Applied Meteorology 1993; 32:1669-1682.

Whiteman CD. Mountain Meteorology: Fundamentals and Applications. New York: Oxford University Press; 2000.

World Meteorological Organization - WMO. Wind measurements: Potential wind speed derived from wind speed fluctuations measurements, and the representativity of wind stations (J.P. van der Meulen). In: Proceedings of the WMO Technical Conference on Meteorological and Environmental Instruments and Methods of Observation (TECO-2000); 2000; Geneva. Geneva; 2000. Instruments and Observing Methods Report n. 74, WMO/TD-n. 1028, p. 72.

Yamartino RJ. A comparison of several single pass estimators of the standard deviation of wind direction. Journal Climate Applied Meteorology 1984; 23: 1362-1366. http://dx.doi.org/10.1175/1520-0450(1984)023\%3C1362 :ACOSPE\%3E2.0.CO;2

Zängl G, Egger J, Wirth V. Diurnal Winds in the Himalayan Kali Gandaki Valley. Part II: Modeling. Monthly Weather Review 2001; 129(5): 1062-1079. http://dx.doi.org/10.1175/1520-0493(2001)129\%3C106 2:DWITHK\%3E2.0.CO;2

Zeri M, Oliveira-Júnior JF, Lyra GB. Spatiotemporal analysis of particulate matter, sulfur dioxide and carbon monoxide concentrations over the city of Rio de Janeiro, Brazil. Meteorology and Atmospheric Physics (Print) 2011; 113: 1-14. http://dx.doi.org/10.1007/s00703-0110153-9 